\title{
REVIEW
}

\section{Pharmacological principles guiding prolonged glucocorticoid treatment in ARDS}

\author{
Gianfranco Umberto Meduri ${ }^{1,8^{*}}$ (D, Djillali Annane ${ }^{2}$, Marco Confalonieri ${ }^{3}$, George P. Chrousos ${ }^{4}$, \\ Bram Rochwerg ${ }^{5,9}$, Amanda Busby $^{6}$, Barbara Ruaro ${ }^{3}$ and Bernd Meibohm ${ }^{7}$
}

(c) 2020 This is a U.S. government work and not under copyright protection in the U.S.; foreign copyright protection may apply

\begin{abstract}
Current literature addressing the pharmacological principles guiding glucocorticoid (GC) administration in ARDS is scant. This paucity of information may have led to the heterogeneity of treatment protocols and misinterpretation of available findings. GCs are agonist compounds that bind to the GC receptor (GR) producing a pharmacological response. Clinical efficacy depends on the magnitude and duration of exposure to GR. We updated the meta-analysis of randomized trials investigating GC treatment in ARDS, focusing on treatment protocols and response. We synthesized the current literature on the role of the GR in GC therapy including genomic and non-genomic effects, and integrated current clinical pharmacology knowledge of various GCs, including hydrocortisone, methylprednisolone and dexamethasone. This review addresses the role dosage, timing of initiation, mode of administration, duration, and tapering play in achieving optimal response to GC therapy in ARDS. Based on RCTs'findings, GC plasma concentration-time profiles, and pharmacodynamic studies, optimal results are most likely achievable with early intervention, an initial bolus dose to achieve close to maximal GRa saturation, followed by a continuous infusion to maintain high levels of response throughout the treatment period. In addition, patients receiving similar GC doses may experience substantial between-patient variability in plasma concentrations affecting clinical response. GC should be doseadjusted and administered for a duration targeting clinical and laboratory improvement, followed by dose-tapering to achieve gradual recovery of the suppressed hypothalamic-pituitary-adrenal (HPA) axis. These findings have practical clinical relevance. Future RCTs should consider these pharmacological principles in the study design and interpretation of findings.
\end{abstract}

Keywords: Acute respiratory distress syndrome, Glucocorticoid, Randomized trial, Glucocorticoid receptor, Duration, Receptor affinity, Pharmacodynamic

\footnotetext{
*Correspondence: gmeduri@uthsc.edu

${ }^{8}$ Pulmonary, Critical Care, and Sleep Medicine Service and Research

Service, Memphis Veterans Affairs Medical Center, 1030 Jefferson Avenue,

Suite room \#CW444, Memphis, TN 38104, USA

Full author information is available at the end of the article
}

\section{重




\section{Introduction}

The results of recently published randomized controlled trials (RCTs) [1,2] provide more robust evidence supporting the efficacy and safety of prolonged glucocorticoid (GC) treatment in acute respiratory distress syndrome (ARDS), despite the heterogeneity in etiologies and severity. GCs are agonist compounds that bind to the ligand-binding domain of the glucocorticoid receptor (GR) to produce a biological (or pharmacological) response. Most anti-inflammatory effects of GCs are mediated through the GR $\alpha$ isoform. The rationale for GC treatment in ARDS is similar to the one for severe coronavirus disease 2019 (COVID-19) [3] and is reviewed in the Digital Supplement 1. Prolonged ( $\geq 7$ days) lowto-moderate dose GC treatment is an intervention directed at the core pathogenetic mechanisms of ARDS

\section{Take-home message}

To date there is scant literature addressing the pharmacological principles guiding glucocorticoid administration in ARDS. This review addresses how the administration mode plays a more significant role in affecting response than the specific molecule. Dosage (initial, overtime, and adjustments based on response), timing of initiation, mode of administration, duration of therapy, tapering, and co-interventions to support the glucocorticoid receptor a function are all essential elements to achieving optimal response to therapy.

(Digital Supplement 1; Table S1), associated with rescue of the cellular concentrations and functions of activated GC-GR $\alpha$ (Fig. 1) and mitochondria [4], exerting a positive effect on all "layers" - cell biology, histology, and physiology - of the disease process, leading to accelerated disease resolution (decreased allostatic load) and improved short- and long-term outcomes [5].
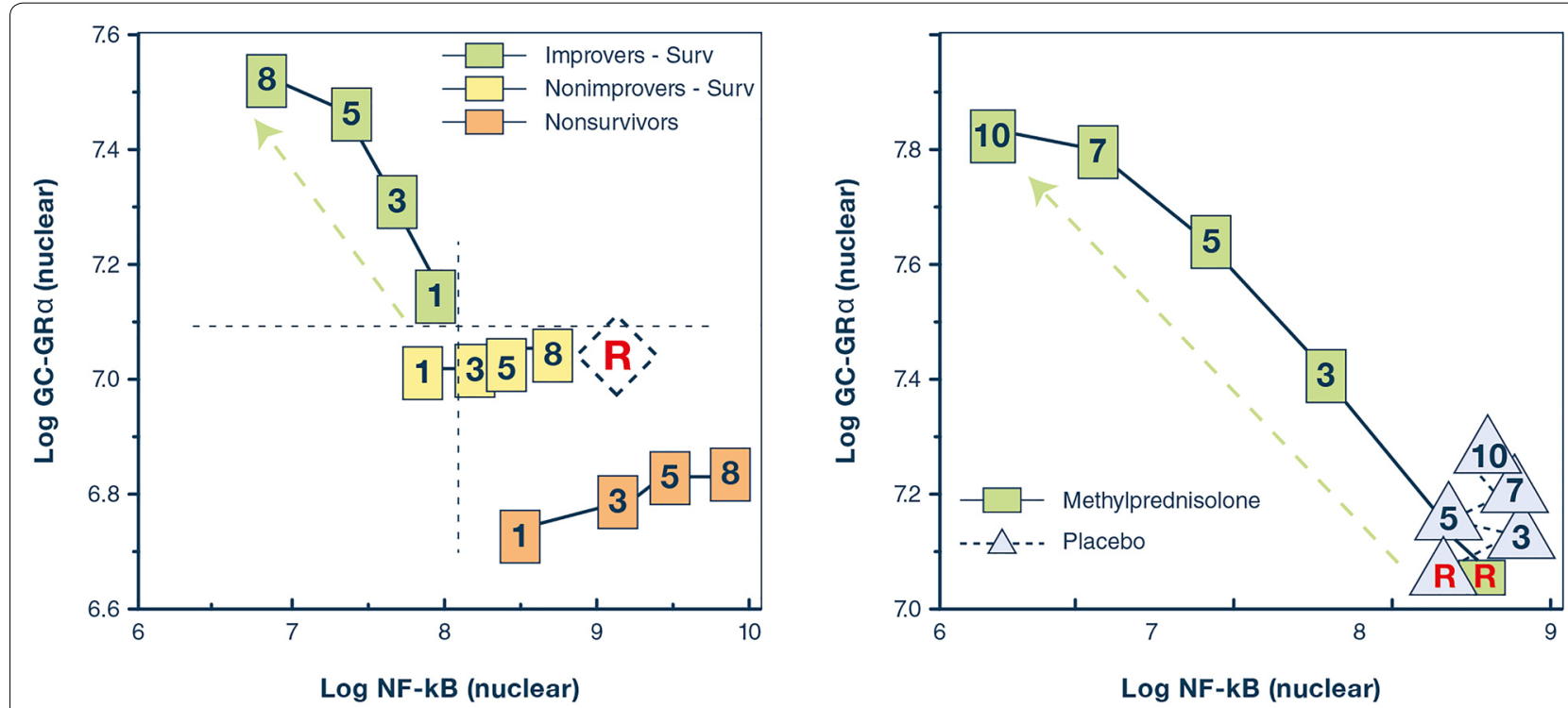

Fig. 1 Relations on natural logarithmic scales between mean levels of nuclear NF-KB and nuclear GC-GRa during the natural progression of ARDS, and in response to prolonged methylprednisolone treatment. Mean intracellular changes of nuclear GC-activated GRa and NF-kB were observed by exposing peripheral blood mononuclear cells of a healthy volunteer to ARDS patient plasma samples collected longitudinally (days 1, 3, 5, and 8) and after randomization to methylprednisolone treatment (randomization day (R) and post-randomization days 3, 5, 7, and 10). The mean values of nuclear NF-KB are plotted against the mean nuclear GC-GRa levels. Improvers had a pre-defined improvement in lung injury score [45] and/or gas exchange component by day 7. The left panel shows ARDS patients with adaptive and maladaptive responses. In improvers, an inverse relationship was observed between these two transcription factors, with the longitudinal direction of the interaction shifting leftwards (decreased NF-KB) and upward (increased GC-GRa). Conversely, in non-improvers, NF-kB increased over time, while GC-GRa showed no significant changes. We defined the first interaction as GC-GRa-driven, and the second interaction as NF-kB-driven [80]. The right panel shows non-improvers-survivors randomized after day 8 of ARDS to methylprednisolone $(n=11)$ vs. placebo $(n=6)$. After natural logarithmic transformation and adjustment for repeated measurements, partial correlations among responses to plasma from the methylprednisolone group were $-0.92(p<0.0001)$ both for nuclear NF-KB and nuclear GRa. For responses to plasma from the placebo group, no significant relationship was found between nuclear NF-KB and nuclear GRa $(r=0.11 ; p=0.70)$ [7]. Prolonged methylprednisolone treatment was associated with upregulation in all measurements of GC-GRa-activity leading to reduction in NF-KB DNA-binding and transcription of inflammatory cytokines. Glucocorticoid treatment changed the longitudinal direction of systemic inflammation from dysregulated (NF-KB-driven, maladaptive response) to regulated (GRa-driven, adaptive response) with significant improvement in indices of alveolar-capillary membrane permeability and markers of inflammation, hemostasis, and tissue repair. Reproduced with permission from reference [5] 
The lack of a well-defined rationale for GC treatment's critical components has resulted in considerable heterogeneity in the protocols investigated in randomized controlled trials (RCTs). While most pharmacological data originate from literature investigating methylprednisolone, we believe that administration mode is more important in affecting response than the specific molecule. The dosage (initial, over time, and adjustments based on response), the timing of initiation, mode of administration, duration, tapering, and co-interventions to support the GR $\alpha$ function are all essential elements to achieving optimal response to therapy. We present a summary of the pharmacological principles that should guide GC treatment in ARDS patients based on results of RCTs, concentration-time profiles for different dosing regimens, and pharmacodynamic studies. This review takes also into consideration recent discoveries (Digital Supplement 1) underscoring the central regulatory function of the activated GR $\alpha$ (GC-GR $\alpha)$ in critical illness throughout disease development and resolution (Fig. 2) [4].

\section{Pharmacological principles of glucocorticoid actions}

Binding affinity and potency

The magnitude of the in vitro response to the GC drug is proportional to the number of GR complexes, and the binding affinity to the receptor, and is characterized by the maximum response a drug is able to produce [6]. The concentration of GR $\alpha$ in cells can be increased by exogenous GC administration (Digital Supplement 1) [7]. Binding affinity is expressed as relative receptor affinity (RRA), and dexamethasone is frequently the reference point for RRA with an arbitrary RRA value of 100 . The RRAs for hydrocortisone, methylprednisolone, and dexamethasone are 9,42 , and 100, respectively [8]. In vivo potency is a measure of the concentration, usually in plasma or serum that is required to produce a drug response, often expressed as concentration required to achieve $50 \%$ of the maximum therapeutic effect $\left(\mathrm{EC}_{50}\right.$ or $\left.\mathrm{IC}_{50}\right)$ [9]. The RRA of GCs is strongly correlated with the inverse of their $\mathrm{IC}_{50}$ for unbound, pharmacologically active concentrations [8]. RRA and $\mathrm{IC}_{50}$ are the corresponding in vitro and in vivo parameters that characterize GC potency and are specific to a GC agonist molecule. However, drug effects in vivo are the result of the interplay

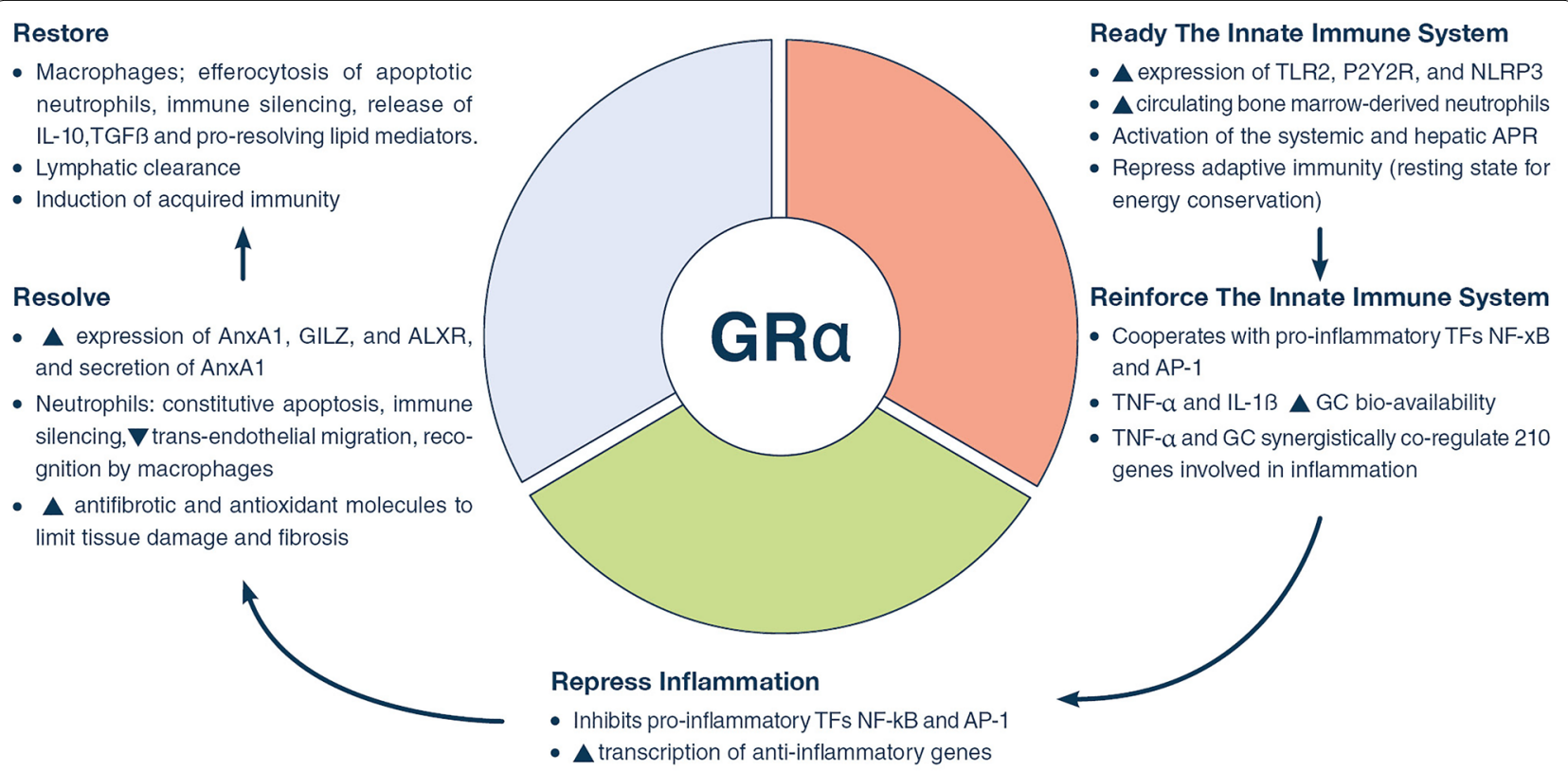

Fig. 2 Glucocorticoid receptor a as a cellular rheostat of homeostatic corrections. The glucocorticoid receptor a (GRa) acts as a cellular rheostat to ensure that a proper response is elicited by the neuroendocrine and immune systems throughout the three phases of homeostatic corrections. The serial sequence of regulatory functions includes the: (1) activation and reinforcement of innate immunity (ready-reinforce), (2) downregulation of pro-inflammatory transcription factors (repress), and (3) promotion of disease resolution (resolve- restore).by switching production from proinflammatory to pro-resolving mediators, while at the same stimulating antifibrotic and antioxidant molecules that limit tissue damage and fibrosis, to help achieve optimal restoration of anatomy and function of the affected tissues, and (4) parallel support of adaptive immunity. Modified with permission from reference.[4]. TLR2 toll-like receptor 2, purinergic receptor P2Y2R; NLRP3 NOD-like receptor pyrin containing 3, APR acute phase response, TF transcription factor, NF-KB nuclear factor-KB, AP-1 activator protein-1, AnxA1 annexinA1, AnxA1 receptor, ALXR A4 lipoxin receptor, GILZ glucocorticoid-induced leucine zipper, TGF $\beta$ transforming growth factor $\beta$ 
of pharmacokinetics, i.e., the time course of the effective $\mathrm{GC}$ concentrations at the target site produced by the applied dose strength and dosing regimens, and pharmacodynamics, i.e., the GC's concentration-effect relationship determined by its potency. Thus, a lower in vitro GC potency (i.e. low RRA or correspondingly high $\mathrm{IC}_{50}$ ) can be offset clinically by higher and/or more frequent doses, providing higher target site concentrations.

The genomic, non-genomic and mitochondrial GC signaling pathways are shown in the Digital Supplement 1 (Figure S1) [4]. The GC genomic effects become evident as early as $30 \mathrm{~min}$ after cytosolic GR $\alpha$ binding to positive (transactivation) or negative (cis repression) GC response elements (GREs) in nuclear DNA, as well as to mitochondrial DNA GREs (mtGRE). Moreover, the GC-GR $\alpha$ complex interacts with other transcription factors, such as nuclear factor- $\mathrm{kB}(\mathrm{NF}-\mathrm{kB})$ or activator protein-1 (AP-1) and alters their transcriptional activities. A recent review reported on the $\mathrm{GC}$ regulation of mitochondrial transcription, via activation of mtGRE [10]. The degree of cytosolic GR $\alpha$ saturation is a direct modulator of the intensity of (therapeutic) GC genomic effects [11]. Almost complete receptor saturation can be achieved by a methylprednisolone-equivalent single dose of 80-100 mg [11]. Specific non-genomic effects (not mediated by induction or repression of specific genes) of GCs take a few minutes to be expressed, are mediated by plasma membrane glucocorticoid receptors, and require higher GC concentrations. Rapid non-genomic actions include, anti-inflammatory regulation, e.g., inhibition of neutrophil degranulation and macrophage superoxide anion production, and vasomotor regulation, e.g., potentiation of systemic response to norepinephrine and local induction of endothelial nitric oxide [12,13].

Since GR $\alpha$ ultimately controls GC-mediated activity, any condition affecting its concentration, binding affinity, transport to the nucleus, GREs interaction in the cell nucleus and mitochondria, cofactor activity, oxidative stress, or interaction with other relevant transcription factors (NF-kB; AP-1) and co-regulators, may affect the cell response to GCs $[14,15]$. Recent reviews have investigated the various ways the pro-inflammatory environment of critical illness can negatively influence GR $\alpha$ function [15-17]. It is noteworthy that the number and function of the activated GR $\alpha$ can be modulated by the administration of exogenous GCs and other co-interventions to improve cellular responsiveness [4]. Randomized studies $[7,18,19]$ suggested that quantitatively adequate and prolonged GC supplementation increased GR $\alpha$ number and action in both circulating and tissue cells, reversing critical illness-related corticosteroid insufficiency (Digital Supplement 1; Table S1).

\section{Determinants of clinical efficacy}

Clinical efficacy depends on both pharmacodynamics (i.e., potency) and pharmacokinetics [i.e., exposure (=magnitude and duration) of the drug at the receptor site]. Together, both processes determine efficacy: potency + presence at the receptor site $\rightarrow$ momentary effect course (effect duration) $\rightarrow$ sum of momentary effects $\rightarrow$ clinical efficacy [20]. Mathematically, pharmacokinetic/pharmacodynamic models can be applied to describe the momentary effect-time course that stem from a specific GC dosing regimen. This effect-time course is characterized by the duration of effect (i.e., the time until the effect drops below a specified value), as well as peak magnitude. The momentary effect-time course can be summed up as the area under the effecttime curve (AUETC or AUEC) or as the area between the baseline and effect curve (ABEC), which drive the clinical effect [20]. The time to reach a pharmacokinetic steady state is determined by the elimination half-life of the drug.

As most pharmacological studies evaluating GCs come from the rheumatology literature, the currently used genomic potency of natural and synthetic GCs is mainly based on lymphocyte proliferation suppression assays and may not be necessarily applicable to critical care [21]. Thus, a re-evaluation of genomic potency based on inhibition of pro-inflammatory proteins is needed. Dose-response studies evaluating potencies for genomic and non-genomic GC effects reported different values (Digital Supplement 1; Table S2). Non-genomic activities related to prostaglandin (PG) $\mathrm{E}_{2}$ inhibition, and arachidonic acid release through suppression of phospholipase A2 synthesis were studied in 549 epithelial cell cultures. The cells first stimulated with IL- $1 \beta$ and then incubated for $3 \mathrm{~h}$ with different GC molecules [22]. Contrary to the classic genomic responses, epithelial cells exposed to methylprednisolone had a higher non-genomic response than those exposed to dexamethasone.

\section{Dose-response}

It has been reported that GCs have a substantially higher potency for genomic than non-genomic effects. Therefore, genomic effects are usually predominant at low GC doses. Increasing GC doses allows the genomic expression modulation to reach a maximum saturation level quickly and then plateau off, so that further dose increases may largely be limited to only extending the duration rather than the intensity of genomic expression modulation. Depending on how genomic expression modulation is transduced into specific biomarker or clinical effects, GC dose increases beyond that of saturation level may lead to only limited further gain in some genomic effects at the biomarker or clinical level, 
but may still increase in others [23]. Conversely, nongenomic effects are usually triggered at substantially higher doses than genomic effects and intensify in line with the increasing dose until leveling off at high-dose therapy. Consequently, the total clinical effect as the sum of genomic and non-genomic effects demonstrate continuous dose-dependent increases despite saturation of genomic expression modulation at moderate GC dose levels. However, the exact relationship between GC dose, cellular concentrations and clinical effects remains to be established, especially for non-genomic effects.

\section{ARDS randomized trials: individual concentration-time courses}

Various GCs and dosing regimens have been used to treat ARDS. The treatment protocols used in ten randomized controlled trials (RCTs) investigating prolonged GC treatment in patients with early ( $\leq 72 \mathrm{~h}$ ) or late ( $\geq 7$ days) ARDS is shown in Digital Supplement 1 (Table S3). This analysis examined methylprednisolone $(n=322)$ [24-28], hydrocortisone $(n=494)$ [29-32], and dexamethasone $(n=277)$ [1], for a duration of therapy between 7 and 32 days. These RCTs reported that GC treatment was associated with a consistent improvement in systemic inflammation markers and oxygenation indices, and an important reduction in duration of mechanical ventilation (MV) and intensive care unit stay (Digital Supplement 1; Table S4).

The corresponding average GC plasma concentration-time profiles expressed as methylprednisolone equivalents based on RRA are shown in Fig. 3. The representation reveals substantial differences among the different GC dosing regimens with regard to exposure. Indeed, infrequent dosing (with dosing intervals of 8 or $24 \mathrm{~h}$ ) leads to substantial time periods without relevant GC serum exposure where the maintenance of GC effects in the target organs and tissues would be dependent on either persistent GC local exposure in those effect sites, or on mechanisms of temporal persistence of the GC effects beyond those periods of relevant effect site exposure. Conversely, continuous administration via infusion maintains high exposure levels throughout the day. The potential disadvantage regarding the delayed onset of high exposure seen with infusions can be corrected with pre-administration of an adequate loading dose (e.g., methylprednisolone $1 \mathrm{mg} / \mathrm{kg}$ loading dose followed by a $1 \mathrm{mg} / \mathrm{kg} /$ day infusion). For non-genomic effects, it is important that GC exposures in treatment regimens are maintained at continuously high exposure levels.

While the above simulations are based on average pharmacokinetic parameters in healthy individuals, GC elimination may be impaired in ARDS patients due to the well-described downregulation of drug metabolizing enzymes in generalized acute inflammation [33]. Yates et al. reported that ARDS patients had an on average $\sim 50 \%$ reduced clearance for methylprednisolone compared to normal values at therapy onset, which returned to normal values with reduction of systemic inflammation within a few days [34]. Correspondingly, methylprednisolone plasma concentrations were elevated relative to healthy individuals in the early treatment phase (Fig. 4). Consequently, the exposure profiles depicted in Fig. 3 may be viewed as a worst-case scenario, i.e. lowest expected exposure for GC therapy in ARDS patients.

\section{Glucocorticoid administration}

Factors affecting prolonged GC treatment response in ARDS are described in Digital Supplement 1 (Figure S2). There is no clear definition for the quantitation of GC doses used in critical illness. In most critical care studies, the hydrocortisone daily equivalent of 400 [35] -500 [36] mg (methylprednisolone 80-100 mg, dexamethasone $15-18.8 \mathrm{mg}$ ) is used to separate low from moderate doses. Based on this definition, the early ARDS studies shown in the Digital Supplement 1 (Table S3), investigated low-to-moderate dose GC treatment. A daily hydrocortisone equivalent of $1500 \mathrm{mg}$ (methylprednisolone $300 \mathrm{mg}$, dexamethasone $56.3 \mathrm{mg}$ ) may be considered a high dose, and between 500 and $1500 \mathrm{mg}$ hydrocortisone equivalent a moderate dose. Most of the data examining the effect of timing, duration, and tapering is extrapolated from individual patient data (IPD) meta-analysis (IPDMA) of four small-to-moderate size

\footnotetext{
(See figure on next page.)

Fig. 3 Concentration-time profiles for different dosing regimens that have been used in the treatment of early ARDS. Glucocorticoid average plasma concentrations were simulated on the basis of published pharmacokinetic parameters for hydrocortisone (HC), methylprednisolone (MP) and dexamethasone (DX) and converted to MP equivalent concentrations using their reported relative receptor affinity (RRA) and fraction unbound to plasma proteins $\left(f_{u}\right)[8,9]$. Pharmacokinetic parameters used for the simulations were as previously described: Clearance 18,21 , and 17 L/h; volume of distribution 33, 64, and $103 \mathrm{~L}$ for HC, MP and DX, respectively. The conversion to MP equivalent concentrations for HC and DX was performed according to the relationship: MP equivalent plasma concentration $=H C$ or DX concentration $\times\left(f_{u, H C}\right.$ or $\left.D X / f_{u, M P}\right) \times\left(R R A_{H C}\right.$ or DX $\left./ R R A_{M P}\right)$. The applied value for RRA and $f_{u}$ were $9,42,100$ and 0.20, 0.23, 0.32 for HC, MP and DX, respectively, as previously reported $[8,9] . f_{u}$ determines the fraction of the drug concentration that is not bound to plasma proteins and is thus available to enter cells and interact with GC receptors, i.e., the fraction of the concentration that is pharmacologically active
} 

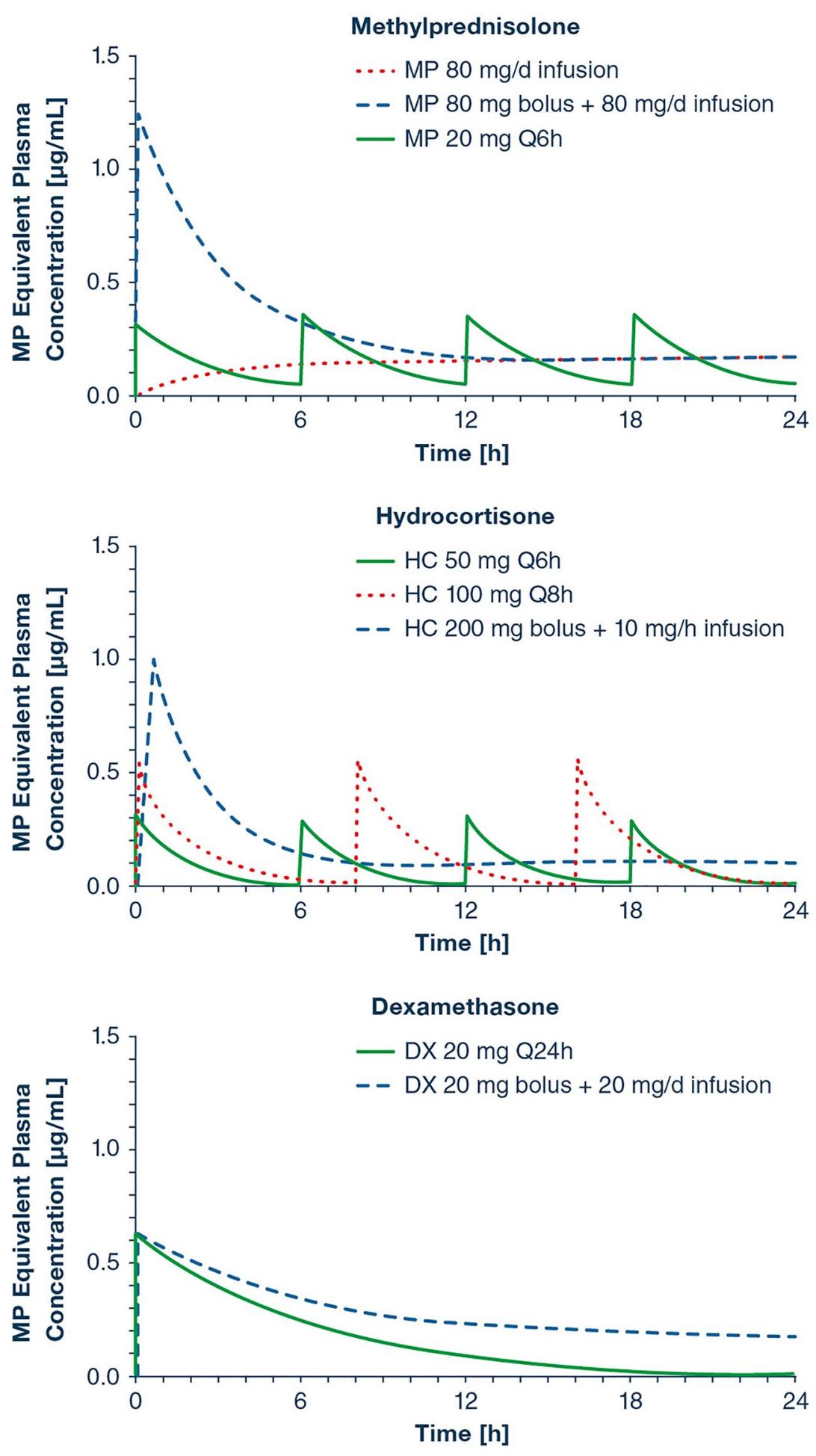

RCTs $(n=322)$ investigating methylprednisolone administration in early and late ARDS [28]. There is a potential therapeutic advantage in treating pulmonary disorders with drugs that have a high penetration into affected lung tissue (Digital Supplement 1). 

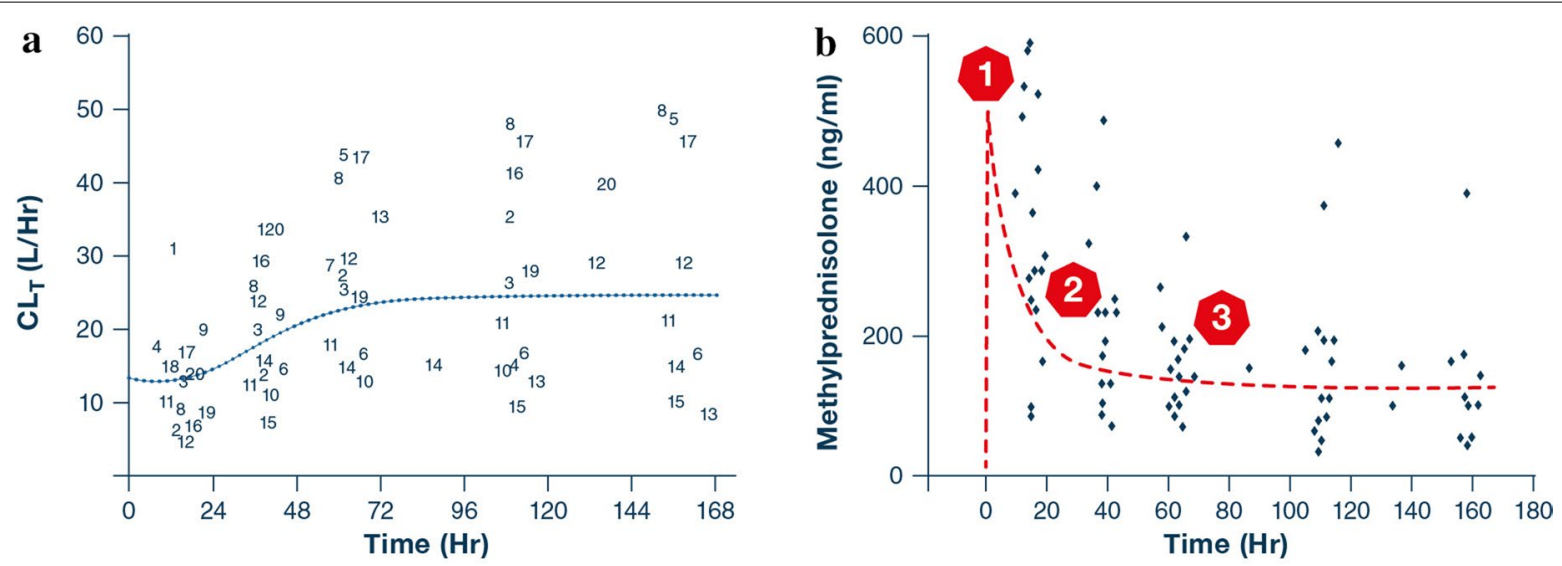

Fig. 4 Methylprednisolone pharmacokinetics in ARDS patients. a Time-dependent increase in methylprednisolone clearance [CLt (L/Hr)] in patients treated with a $1 \mathrm{mg} / \mathrm{kg}$ loading dose, followed by a $1 \mathrm{mg} / \mathrm{kg} /$ day continuous infusion. The high systemic inflammatory state may be responsible for the impaired methylprednisolone metabolism observed in early ARDS at the beginning of therapy. High levels of pro-inflammatory cytokines are known to inhibit the expression and activity of hepatic drug metabolizing enzymes, including multiple cytochrome P450 isozymes relevant for the metabolism of methylprednisolone. Inflammation resolves progressively during continuous therapy, leading to re-establishment of homeostatic conditions of drug metabolizing enzyme systems. This is reflected by a time-dependent increase in methylprednisolone clearance. It took about 2 days $(41.1$ h) of methylprednisolone therapy to achieve $50 \%$ of the improvement in clearance towards the re-establishment of homeostasis.[34]. b Methylprednisolone plasma concentration-time profile in ARDS patients receiving the aforementioned dosing regimen. Methylprednisolone clearance is impaired during time period 1 and its concentrations are high enough to trigger genomic and non-genomic GC effects. This most likely establishes initial control of the generalized inflammatory state [33]. Inflammatory control is at least partially established during period 2 within 2 days of therapy, leading to an increased hepatic methylprednisolone clearance, secondary to re-established drug metabolizing activity. The concentrations are maintained around $203 \pm 147 \mathrm{ng} / \mathrm{mL}$ during period 3, exerting prolonged sustained anti-inflammatory activity. Reproduced with permission from Yates et al. [34]. Importantly, ARDS patients receiving similar GC doses experience a substantial variability in the resulting plasma concentrations due to between-patient variability; this may affect nati-inflammatory response to treatment

In 2017, the Corticosteroid Guideline Task Force of the Society of Critical Care Medicine (SCCM) and the European Society of Intensive Care Medicine (ESICM) released guidelines for GC treatment in critically ill patients [35]. The Task Force applied the Grading of Recommendations Assessment, Development and Evaluation (GRADE) and the Evidence-to-Decision tool making a conditional recommendation for GC treatment providing an on-line detailed treatment protocol (Supplemental Digital Content 5, https://links.lww.com/CCM/C918) (Fig. 5) [35].

\section{Timing}

Early initiation (preferably within $6 \mathrm{~h}$ of diagnosis) of GC treatment, before homeostatic corrections reach exhaustion [4] is critical in decreasing the acute and long-term negative impact of the allostatic load imposed during vital organ support. In the IPDMA, early $(<72 \mathrm{~h})$ as compared to late ( $\geq 7$ days) initiation of methylprednisolone treatment-when fibroproliferation is still in the early development stage (cellular with predominant type III procollagen) - was associated with faster disease resolution, determined by time to extubation [hazard ratio (HR) 3.48; 95\% confidence interval (CI) $2.07-5.85$ in the early group vs. $\mathrm{HR}=2.06$; $95 \% \mathrm{CI} 1.44-2.95$ in late group] and
ICU discharge, despite a lower daily methylprednisolone dose ( $1 \mathrm{mg} / \mathrm{kg} /$ day vs. $2 \mathrm{mg} / \mathrm{kg} /$ day) [28]. Noteworthy, in the early ARDS RCTs, methylprednisolone was administered as a continuous infusion. Delaying treatment and only administering to patients that fail to improve by the third day of ARDS, as proposed by some investigators, may miss this important window to maximize treatment benefit.

\section{Initial dosage}

An adequate initial loading bolus is required, particularly when glucocorticoids are administered as a continuous infusion, to achieve prompt elevation of plasma GC levels and approach maximal saturation of GC receptors (approximately $100 \mathrm{mg}$ of methylprednisolone equivalent) [11] in the cytoplasm and on the cell membrane for genomic and non-genomic actions. A methylprednisolone dose of $1 \mathrm{mg} / \mathrm{kg} / \mathrm{day}$ in early ARDS is similar to what is commonly used in other forms of interstitial lung diseases [37], and close to the dexamethasone dose (20 mg) used in the recent DEXA-ARDS RCT [1]. Experimental [38] and clinical research $[39,40]$ suggest that pulse doses of methylprednisolone $(1000 \mathrm{mg} /$ day $)$ is not beneficial in ARDS. 


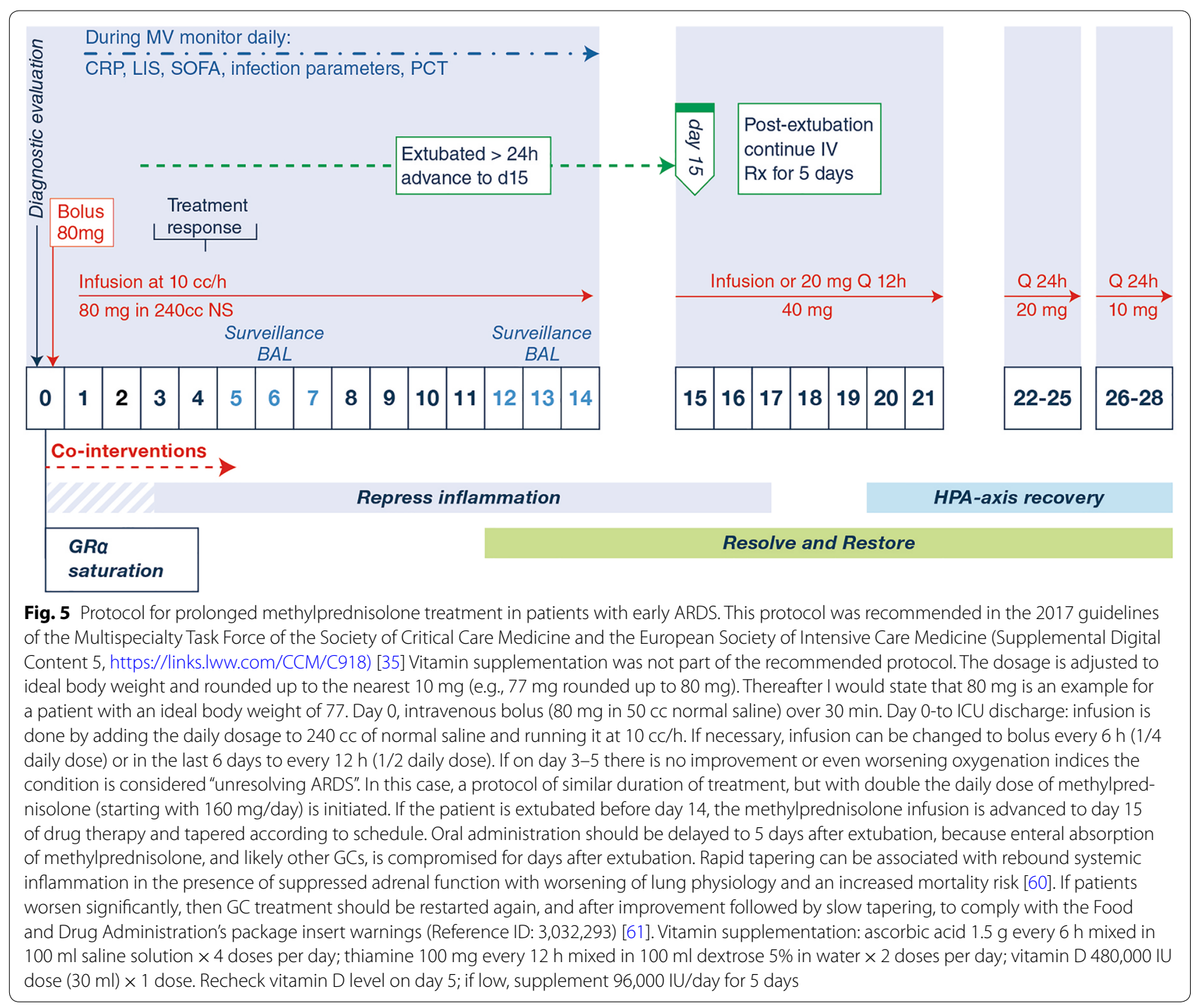

An in vitro study commented positively on the impact GC dosage has on inflammation downregulation. In human monocytic cells activated with graded concentrations of lipopolysaccharide (LPS) and then exposed to increasing concentrations of methylprednisolone (Digital Supplement 1; Figure S3), the reduction in inflammatory cytokine gene (TNF- $\alpha$, IL-1 $\beta$, and IL-6) transcription (irrespective of baseline inflammation severity) was initially modest. It then reached an inflection point, followed by a rapid reduction, most likely related to achieving close to maximal drug receptor saturation for a measurable genomic and non-genomic effect [41]. This finding emphasizes the relevance of adequate dose selection in achieving GR $\alpha$ saturation and optimal results. In one RCT [42], the response to hydrocortisone treatment, based on illness severity, provides some support for this concept.

\section{Mode of administration}

The concentration-time profiles in Fig. 3 suggest that optimal GR exposure can be achieved with an initial loading bolus followed by a continuous infusion (daily dose over $24 \mathrm{~h}$ ) to maintain high response levels throughout the treatment period. Limited data show that RCTs that implemented this administration method [26, 27, 29, 31], vs. RCTs that used intermittent bolus dosing, achieved quicker disease resolution with more mechanical ventilation (MV)-free days (Digital Supplement 2; Figures S1). In patients with septic shock ( 3 RCTs; $n=310$ ), there is no evidence for a mortality benefit between continuous infusion and intermittent bolus [43].

\section{Duration of glucocorticoid administration}

Duration of GC administration and tapering are main determinants of treatment efficacy. The role of GC treatment in ARDS and critical illness is to support the central 
regulatory function played by the activated GR $\alpha$ in the acute phase (anti-inflammatory action) of the disease and throughout the fundamental albeit underappreciated phase of resolution [4]. The resolution phase is associated with multiple biochemical pathways, including switching production from pro-inflammatory to pro-resolving mediators, while also producing antifibrotic and antioxidant proteins that limit tissue damage and fibrosis to achieve optimal restoration of anatomy and function [4, 44].

It is essential to monitor response to treatment during intervention with daily assessment of lung [lung injury score (LIS) [45] and minute ventilation] and multiple organ function [Sequential Organ Failure Assessment (SOFA) score [46]] along with systemic inflammation markers [i.e., C-reactive protein (CRP)]. It sometimes takes two days before a CRP reduction is observed, as, initially, the GCs potentiate innate immunity (Fig. 2) [4]. Importantly, ARDS patients receiving similar GC doses experience a substantial variability in the resulting plasma concentrations due to between-patient variability and additional disease effects on the GC pharmacokinetics, and this may affect clinical response (Fig. 4) [34]. Two RCTs tailored dosage and duration of therapy based on individual patient's response $[24,26]$. If no improvement was observed by day 5 or the condition worsened before then (Fig. 5), treatment was reset to day 1, doubling of the daily dose (i.e., from 80 to $160 \mathrm{mg}$ methylprednisolone), including a new loading bolus [26]. Conversely, if the patient was successfully extubated (off MV $>24 \mathrm{~h}$ ) before day 14 , treatment was advanced to day 15 . After extubation, oxygen requirements provide a simple modality to assess restoration of lung function. One pharmacokinetic study reported that intestinal methylprednisolone absorption is compromised for about 5 days after extubation [34], and that is why switching to oral administration 5 days after MV removal may be a reasonable course of action. Figure 5 provides a summary of our suggested approach for methylprednisolone treatment.

Dexamethasone is an effective treatment in ARDS, as seen in the recently published DEXA-ARDS RCT [1] which examined moderate-to-severe ARDS and the RECOVERY RCT evaluating patients with COVID19-related ARDS [47]. A potential advantage of dexamethasone over alternative GCs is the single daily administration. The DEXA-ARDS treatment regimen provides a satisfactory alternative to the methylprednisolone protocol. However, we believe there may be a potential benefit in continuing dexamethasone treatment following extubation and in adding a 6-to-8-day tapering to the regimen (see below). Likewise, trials on hydrocortisone have suggested significant survival benefits in adults with ARDS [29-31]. Taken together these trials suggested that the favorable benefit to risk balance is a class effect.

\section{Infection surveillance}

It is essential to monitor for evidence of infection in the absence of fever during MV. In one RCT, infection surveillance identified $56 \%$ of nosocomial infections in patients without fever [26]. As procalcitonin is a bacterial infection biomarker that remains unaffected by GC treatment it is useful for early identification of infection [48-51], however, there is a lack of studies on ARDS patients receiving GC treatment. Additional infection monitoring parameters include an unexplained increase in minute ventilation $(\geq 30 \%)$ or a worsening of the LIS or SOFA score. Unless contraindicated, surveillance bronchoscopic or non-bronchoscopic bronchoalveolar lavage (BAL) is useful for early identification of ventilator-associated pneumonia and to monitor lung inflammation (neutrophilia).

\section{Tapering}

Although GCs play a critical role in supporting homeostatic corrections, this is achieved at the expense of reversible suppression of the hypothalamic-pituitary-adrenal (HPA) axis. Unfortunately, the risk of GC treatment-associated adrenal suppression in critically ill patients with dysregulated systemic inflammation is underappreciated. The concept that HPA axis suppression is unlikely if GC treatment lasts less than 3 weeks [52] applies only to the outpatient setting and not to critically ill patients. Neither the total nor the highest dose or the GC treatment duration is a significant predictor of HPA axis recovery [53]. In the recent "Reduction in the Use of Corticosteroids in Exacerbated COPD (REDUCE) trial" that compared prednisone administration at $40 \mathrm{mg}$ daily for 5 vs. 14 days, adrenal suppression was detected at hospital discharge and at thirty days in $38 \%$ and $9 \%$ of patients following randomization, respectively. No differences were observed between 5 and 14 days of GC exposure [54]. Similarly to the experimental literature $[55,56]$, critical care RCTs have shown that abrupt GC discontinuation after a 3-14 days of treatment was rapidly followed by a rebound inflammatory response with severe clinical relapses in about one-third of the patients [25, 57-59], and a potential signal for increased mortality [25]. In the Steinberg trial [25], discontinuation of the study drug (administered as intermittent boluses) within $48 \mathrm{~h}$ of successful extubation was associated with clinical relapse in one-quarter of methylprednisolone-treated patients. These patients necessitated a return to MV and did not receive a re-institution of GC treatment. This led to poor outcomes with additional days of MV and a nine-fold increased risk of 60-day mortality $(p=0.001)$, 
as compared to patients that did not return to MV [60]. In patients with sepsis with or without ARDS evidence for clinically significant rebound after termination of corticosteroids without tapering is missing [43]. Gradual tapering may (1) preserve the improvements achieved during GC administration, (2) sustain continuous resolution and restoration of tissue homeostasis, (3) achieve gradual recovery of the suppressed HPA axis, (4) forestall disease relapse from rebound systemic inflammation, and (5) complies with the Food and Drug Administration's package insert warnings (Reference ID: 3032293) [61].

\section{Co-interventions}

The activated GR $\alpha$ interdependence with functional mitochondria and essential vitamin reserves provides a rationale for co-interventions that include prolonged GC treatment in association with rapid correction of hypovitaminosis to improve cellular responsiveness. Oxidative stress has a direct deleterious impact on GR number and function [4]. Three vitamins, i.e. thiamine (vitamin B1), ascorbic acid (vitamin C), and vitamin D, are important for the proper functioning of the GR system and mitochondria, but their reserves are rapidly exhausted in critical illness [62]. A comprehensive list of suggested mechanisms for the efficacy of thiamine, ascorbic acid, and GCs in critical illness, and an updated rationale for the co-intervention were recently reviewed $[4,63]$. The impact Vitamin $C$ has on activated GR $\alpha$ activity is shown in the Digital Supplement 1 (Figure S4) [4]. A recent RCT on patients with sepsis-associated ARDS reported a possible reduction in 28-day all-cause mortality, although as this was a secondary outcome, it was under-powered [64]. However, other potential co-interventions directed at increasing GR expression, e.g., statins [65], melatonin [66], beta-blockers [67], calcium channel blockers [67], or directed at improving mitochondrial function [68-70] have not been investigated in association either with GC treatment in acute illness or alone in chronic critical illness.

\section{Clinical efficacy}

Digital Supplement 2; Figures S3, S4, and S5 report the impact of prolonged GC treatment on the outcomes of duration of mechanical ventilation, length of ICU stay, and hospital mortality (relative risk 0.65 ; $95 \%$ confidence interval 0.50-0.85-number needed to save one life: 7.2). Importantly, the survival benefit observed during hospitalization persisted after hospital discharge with follow-up observations extending up to 60 days [1, 29, 42], 4 months [24], 6 months [25], or one year (limit of measurement) [26]. Survival rates at 2, 6, and 12 months were $76 \%$ vs. $61 \%(p$-value $=0.13), 67 \%$ vs. $46 \%(p$ value $=0.07)$, and $63.5 \%$ vs. $46 \%$ ( $p$ value $=0.13$ ), respectively [26]. In aggregate data from four RCTs in patients $(n=945)$ hospitalized with community-acquired pneumonia, early initiation of GC treatment prevented progression to ARDS $(n=945 ; 0.4 \%$ vs. $3.0 \%$; RR $0.27,95 \%$ CI $0.08,0.87)$ [71]. Recent RCTs in COVID-19 patients provide additional support for prolonged GC treatment in early ARDS. Rationale for GC treatment in severe COVID-19 was recently reviewed [72]. A prospective meta-analysis of seven RCTs investigating critically ill adults with COVID$19(n=1703)$ reported a significant reduction in 28-day mortality (32.7\% vs.41.5\%; OR $0.66,95 \% \mathrm{CI}, 0.53-0.82$ ). There were no differences in survival benefit between the three corticosteroids investigated (dexamethasone for 10 days, hydrocortisone for 7 or 14 days, and methylprednisolone for 5 days), and no evidence for increased risk of serious complications [2]. These findings led the World Health Organization to recommend GC treatment for severe COVID-19 [73].

\section{Therapeutic index}

The therapeutic index is a measure of drug safety. Except for transient hyperglycemia (mostly within $36 \mathrm{~h}$ after an initial bolus), GC treatment is not associated with a higher risk of neuromuscular weakness, [74] gastrointestinal bleeding, or nosocomial infection (Digital Supplement 2; Figure S6). Moreover, GC treatment-associated downregulation of systemic and pulmonary inflammation might lower the risk of developing nosocomial infections by (1) decreasing MV duration, (2) achieving an inflammatory milieu less favorable for intra- and extra- cellular growth of the bacterial pathogens frequently encountered in ARDS (Staphylococcus aureus, Pseudomonas aeruginosa, and Acinetobacter sps.) [75], and (3) improving opsonization-dependent phagocytic neutrophil function [76] and intracellular killing [41]. Inflammation has a bidirectional effect on the growth of nosocomial pathogens; lower inflammatory cytokine levels-similar to values detected in ARDS survivors-suppress growth, whilst higher levels-similar to values detected in ARDS nonsurvivors-enhance bacterial growth in a dosedependent manner [75]. In LPS-activated immune cells exposed to graded doses of methylprednisolone, concentrations similar to the plasma levels $(150-250 \mu \mathrm{g} / \mathrm{ml})$ achieved in ARDS patients on methylprednisolone infusion $(1 \mathrm{mg} / \mathrm{kg} /$ day $)$ [34] were associated with the most significant reduction in intracellular bacterial growth and TNF- $\alpha$, IL-1 $\beta$, and IL-6 gene expression.[41]

The endothelial GR $\alpha$ is a critical regulator of vascular homeostatic corrections, and essential for restoring the integrity of the blood-brain barrier [4]. In ARDS survivors, longer GC treatment was associated with lower anxiety scores $(p$ value $=0.019)$ and a trend $(p$ value $=0.05)$ for improvement in post-traumatic stress 
syndrome score symptomatology [77], similar to the improvements previously reported in patients with septic shock [78].

\section{Conclusions and future directions}

To the best of our knowledge, this is the first review examining the pharmacological principles guiding GC treatment in ARDS which examines how each component of the treatment protocol is relevant to achieve optimal results. We suggest that future RCTs evaluating GC treatment in ARDS should consider these pharmacological principles in their design and protocol. RCTs comparing how different GC doses and duration of treatment impact on laboratory markers of inflammation and oxygenation, duration of mechanical ventilation, and shortand long-term morbidity and mortality have important clinical relevance. An effort is in progress to best characterize subphenotypes in ARDS [79], to identify those more likely to benefit from an intervention. An approach based on these pharmacological principles may help to eventually personalize GC treatment protocols based on patients' individual clinical and laboratory characteristics with longitudinal adjustments directed by measurement of physiological and laboratory markers.

\section{Electronic supplementary material}

The online version of this article (https://doi.org/10.1007/s00134-020-06289-8) contains supplementary material, which is available to authorized users.

\begin{abstract}
Author details
${ }^{1}$ Memphis Veterans Affairs Medical Center Research Service, Departments of Medicine, University of Tennessee Health Science Center, Memphis, TN, USA ${ }^{2}$ Department of Critical Care, Raymond Poincaré Hospital (AP-HP), Laboratory Infection and Inflammation, U1173, Faculty of Health Science Simone Veil, University of Versailles SQY, University Paris Saclay, INSERM, FHU SEPSIS, RHU RECORDS, Versailles, France. ${ }^{3}$ Pulmonology Department, University Hospital of Cattinara, University of Trieste, Trieste, Italy. ${ }^{4}$ University Research Institute of Maternal and Child Health and Precision Medicine, National and Kapodistrian University of Athens, Medical School, Athens, Greece. ${ }^{5}$ Department of Medicine, McMaster University, Hamilton, ON, Canada. ${ }^{6}$ Health Research Methods Unit, School of Life and Medical Sciences, University of Hertfordshire, Hatfield, UK. ${ }^{7}$ Department of Pharmaceutical Sciences, University of Tennessee Health Science Center, Memphis, TN, USA. ${ }^{8}$ Pulmonary, Critical Care, and Sleep Medicine Service and Research Service, Memphis Veterans Affairs Medical Center, 1030 Jefferson Avenue, Suite room \#CW444, Memphis, TN 38104, USA. ${ }^{9}$ Department of Health Research Methods, Evidence and Impact, McMaster University, Hamilton, ON, Canada.
\end{abstract}

\section{Acknowledgements}

The authors thank Professor William J. Jusko, PhD, University at Buffalo School of Pharmacy and Pharmaceutical Sciences for critical review of the manuscript and Barbara Wade for her linguistic advice.

\section{Author contributions}

GUM, DA, MC, GC, and BM equally contributed to the writing of this manuscript, $B R$, and $A B$ contributed with an updated meta-analysis of randomized studies investigating prolonged glucocorticoid treatment in ARDS. BR contributed with a review of the pertinent literature and a summary of findings. All authors contributed substantially to editing, revising and finalizing the manuscript before submission. All authors approved the final manuscript.
Funding

This material is the result of work supported with the resources and use of facilities at the Memphis VA Medical Center.

\section{Compliance with ethical standards}

\section{Conflicts of interest}

The authors declare no conflict of interest.

\section{Ethics committee approval}

The contents of this commentary do not represent the views of the U.S. Department of Veterans Affairs or the United States Government.

\section{Publisher's Note}

Springer Nature remains neutral with regard to jurisdictional claims in published maps and institutional affiliations.

Received: 24 September 2020 Accepted: 8 October 2020

Published online: 4 November 2020

\section{References}

1. Villar J, Ferrando C, Martinez D, Ambros A, Munoz T, Soler JA, Aguilar G, Alba F, Gonzalez-Higueras E, Conesa LA, Martin-Rodriguez C, DiazDominguez FJ, Serna-Grande P, Rivas R, Ferreres J, Belda J, Capilla L, Tallet A, Anon JM, Fernandez RL, Gonzalez-Martin JM, dexamethasone in An (2020) Dexamethasone treatment for the acute respiratory distress syndrome: a multicentre, randomised controlled trial. Lancet Respir Med 8:267-276

2. Group WHOREAfC-TW, Sterne JAC, Murthy S, Diaz JV, Slutsky AS, Villar J, Angus DC, Annane D, Azevedo LCP, Berwanger O, Cavalcanti AB, Dequin PF, Du B, Emberson J, Fisher D, Giraudeau B, Gordon AC, Granholm A Green C, Haynes R, Heming N, Higgins JPT, Horby P, Juni P, Landray MJ, Le Gouge A, Leclerc M, Lim WS, Machado FR, McArthur C, Meziani F, Moller MH, Perner A, Petersen MW, Savovic J, Tomazini B, Veiga VC, Webb S,Marshall JC, (2020) Association between administration of systemic corticosteroids and mortality among critically III patients with COVID19: a meta-analysis. JAMA 324(13):1330-1341. https://doi.org/10.1001/ jama.2020.17023

3. Park JH, Lee HK (2020) Re-analysis of single cell transcriptome reveals that the NR3C1-CXCL8-neutrophil axis determines the severity of COVID-19. Front Immunol 11:2145

4. Meduri GU,Chrousos GP, (2020) General adaptation in critical illness: glucocorticoid receptor-alpha master regulator of homeostatic corrections. Front Endocrinol (Lausanne) 11:161. https://doi.org/10.3389/fendo 2020.0016

5. Meduri GU, Annane D, Chrousos GP, Marik PE, Sinclair SE (2009) Activation and regulation of systemic inflammation in ARDS: Rationale for prolonged glucocorticoid therapy. Chest 136:1631-1643

6. Stringer J (2017) Basic concepts in pharmacology: what you need to know for each drug class. In: Editor (eds) Book Basic concepts in pharmacology: what you need to know for each drug class. McGraw Hill Professional

7. Meduri GU, Tolley EA, Chrousos GP, Stentz F (2002) Prolonged methylprednisolone treatment suppresses systemic inflammation in patients with unresolving acute respiratory distress syndrome. Evidence for inadequate endogenous glucocorticoid secretion and inflammation-induced immune cell resistance to glucocorticoids. Am J Respir Crit Care Med 165:983-991

8. Derendorf H, Möllmann H, Hochhaus G, Meibohm B, Barth J (1997) Clinical PK/PD modelling as a tool in drug development of corticosteroids. Int J Clin Pharmacol Ther 35:481-488

9. Derendorf H, Hochhaus G, Mollmann H, Barth J, Krieg M, Tunn S, Mollmann C (1993) Receptor-based pharmacokinetic-pharmacodynamic analysis of corticosteroids. J Clin Pharmacol 33:115-123

10. Lapp HE, Bartlett AA, Hunter RG (2019) Stress and glucocorticoid receptor regulation of mitochondrial gene expression. J Mol Endocrinol 62:R121-R128 
11. Buttgereit F, da Silva JA, Boers M, Burmester GR, Cutolo M, Jacobs J, Kirwan J, Kohler L, Van Riel P, Vischer T, Bijlsma JW (2002) Standardised nomenclature for glucocorticoid dosages and glucocorticoid treatment regimens: current questions and tentative answers in rheumatology. Ann Rheum Dis 61:718-722

12. Jiang CL, Liu L, Tasker JG (2014) Why do we need nongenomic glucocorticoid mechanisms? Front Neuroendocrinol 35:72-75

13. Charmandari E, Nicolaides NC, Chrousos GP (2014) Adrenal insufficiency. Lancet 383:2152-2167

14. Dendoncker K, Libert C (2017) Glucocorticoid resistance as a major drive in sepsis pathology. Cytokine Growth Factor Rev 35:85-96

15. Dejager L, Vandevyver S, Petta I, Libert C (2014) Dominance of the strongest: inflammatory cytokines versus glucocorticoids. Cytokine Growth Factor Rev 25:21-33

16. Meduri GU, Yates CR (2004) Systemic inflammation-associated glucocorticoid resistance and outcome of ARDS. Ann NY Acad Sci 1024:24-53

17. Annane D, Pastores S, Arlt W, Balk R, Beishuizen A, Briegel J, Carcillo J, Christ-Crain M, Cooper M, Marik P (2017) Critical illness-related corticosteroid insufficiency (CIRCI): a narrative review from a multispecialty task force of the society of critical care medicine (SCCM) and the European Society of Intensive Care Medicine (ESICM). Crit Care Med 45:2089-2099

18. Wang XQ, Zhou X, Zhou Y, Rong L, Gao L, Xu W (2008) Low-dose dexamethasone alleviates lipopolysaccharide-induced acute lung injury in rats and upregulates pulmonary glucocorticoid receptors. Respirology 13:772-780

19. Zhang $Y$, Zuo W, Rong Q, Teng G, Y Z, (2010) Glucocorticoid receptor expression on acute lung injury induced by endotoxin in rats. World J Emerg Med 1:65-69

20. Czock D, Keller F, Rasche FM, Haussler U (2005) Pharmacokinetics and pharmacodynamics of systemically administered glucocorticoids. Clin Pharmacokinet 44:61-98

21. Mollmann H, Balbach S, Hochhaus G, Barth J,Derendorf H, (1995) Pharmacokinetic-pharmacodynamic correlations of corticosteroids. Handbook of pharmacokinetic/pharmacodynamic correlation: 323-362

22. Croxtall JD, Van Hal PTW, Choudhury Q, Gilroy DW, Flower RJ (2002) Different glucocorticoids vary in their genomic and non-genomic mechanism of action in A549 cells. Br J Pharmacol 135:511-519

23. Ayyar VS, Jusko WJ (2020) Transitioning from basic toward systems pharmacodynamic models: lessons from corticosteroids. Pharmacol Rev 72:414-438

24. Meduri GU, Headley AS, Golden E, Carson SJ, Umberger RA, Kelso T, Tolley EA (1998) Effect of prolonged methylprednisolone therapy in unresolving acute respiratory distress syndrome: a randomized controlled trial. JAMA 280:159-165

25. Steinberg KP, Hudson LD, Goodman RB, Hough CL, Lanken PN, Hyzy R, Thompson BT, Ancukiewicz M, National Heart L, Blood Institute Acute Respiratory Distress Syndrome Clinical Trials N (2006) Efficacy and safety of corticosteroids for persistent acute respiratory distress syndrome. N Engl J Med 354:1671-1684

26. Meduri GU, Golden E, Freire AX, Taylor E, Zaman M, Carson SJ, Gibson M, Umberger R (2007) Methylprednisolone infusion in early severe ARDS: results of a randomized controlled trial. Chest 131:954-963

27. Rezk N, Ibrahim A (2013) Effects of methylprednisolone in early ARDS. Egypt J Chest Dis Tuberc 62:167-172

28. Meduri GU, Bridges L, Shih MC, Marik PE, Siemieniuk RAC, Kocak M (2016) Prolonged glucocorticoid treatment is associated with improved ARDS outcomes: analysis of individual patients' data from four randomized trials and trial-level meta-analysis of the updated literature. Intensive Care Med 42:829-840

29. Confalonieri M, Urbino R, Potena A, Piattella M, Parigi P, Puccio G, Della Porta R, Giorgio C, Blasi F, Umberger R, Meduri GU (2005) Hydrocortisone infusion for severe community-acquired pneumonia: a preliminary randomized study. Am J Respir Crit Care Med 171:242-248

30. Annane D, Sebille V, Bellissant E, Ger-Inf-05 Study G (2006) Effect of low doses of corticosteroids in septic shock patients with or without early acute respiratory distress syndrome. Crit Care Med 34:22-30

31. Sabry NA, Omar EE-D (2011) Corticosteroids and ICU course of community acquired pneumonia in Egyptian settings. Pharmacol Pharm 2:73-81

32. Liu L, Li J, Huang YZ, Liu SQ, Yang CS, Guo FM, Qiu HB, Yang Y (2012) The effect of stress dose glucocorticoid on patients with acute respiratory distress syndrome combined with critical illness-related corticosteroid insufficiency. Zhonghua Nei Ke Za Zhi 51:599-603

33. Morgan ET (1997) Regulation of cytochromes P450 during inflammation and infection. Drug Metab Rev 29:1129-1188

34. Yates CR, Vysokanov A, Mukherjee A, Ludden TM, Tolley EA, Meduri GU, Dalton JT (2001) Time-variant increase in methylprednisolone clearance in patients with acute respiratory distress syndrome: a population pharmocokinetic study. J Clin Pharmacol 41:1-10

35. Annane D, Pastores S, Rochwerg B, Arlt W, Balk R, Beishuizen A, Briegel J, Carcillo J, Christ-Crain M, Cooper M (2017) Guidelines for the diagnosis and management of critical illness-related corticosteroid insufficiency (CIRCI) in critically III patients (Part I): society of critical care medicine (SCCM) and European Society of Intensive Care Medicine (ESICM). Crit Care Med 45:2078-2088

36. Volbeda M, Wetterslev J, Gluud C, Zijlstra JG, van der Horst IC, Keus F (2015) Glucocorticosteroids for sepsis: systematic review with metaanalysis and trial sequential analysis. Intensive Care Med 41:1220-1234

37. Jantz MA, Sahn SA (1999) Corticosteroids in acute respiratory failure. Am J Respir Crit Care Med 160:1079-1100

38. Teng D, Pang QF, Yan WJ, Zhao Xin W, Xu CY (2013) The harmful effect of prolonged high-dose methylprednisolone in acute lung injury. Int Immunopharmacol 15:223-226

39. Takaki M, Ichikado K, Kawamura K, Gushima Y, Suga M (2017) The negative effect of initial high-dose methylprednisolone and tapering regimen for acute respiratory distress syndrome: a retrospective propensity matched cohort study. Crit Care 21:135

40. Kido T, Muramatsu K, Asakawa T, Otsubo H, Ogoshi T, Oda K, Kubo T, Fujino Y, Matsuda S, Mayumi T (2018) The relationship between high-dose corticosteroid treatment and mortality in acute respiratory distress syndrome: a retrospective and observational study using a nationwide administrative database in Japan. BMC Pulmonary Med $18: 28$

41. Meduri GU, Kanangat S, Bronze M, Patterson DR, Meduri CU, Pak C, Schaberg DR (2001) Effects of methylprednisolone on intracellular bacterial growth. Clin Diagn Lab Immunol 8:1156-1163

42. Tongyoo S, Permpikul C, Mongkolpun W, Vattanavanit V, Udompanturak S, Kocak M, Meduri GU (2016) Hydrocortisone treatment in early sepsis-associated acute respiratory distress syndrome: results of a randomized controlled trial. Crit Care 20:329

43. Annane D, Bellissant E, Bollaert PE, Briegel J, Keh D, Kupfer Y, Pirracchio R, Rochwerg B, (2019) Corticosteroids for treating sepsis in children and adults. Cochrane Database Syst Rev 12(12):CD002243. https://doi. org/10.1002/14651858.CD002243.pub4

44. Busillo JM, Cidlowski JA (2013) The five Rs of glucocorticoid action during inflammation: ready, reinforce, repress, resolve, and restore. Trends Endocrinol Metab: TEM 24:109-119

45. Murray JF, Matthay MA, Luce JM, Flick MR (1988) An expanded definition of the adult respiratory distress syndrome. Am Rev Respir Dis 138:720-723

46. Vincent JL, Moreno R, Takala J, Willatts S, De Mendonca A, Bruining H, Reinhart CK, Suter PM, Thijs LG (1996) The SOFA (Sepsis-related Organ Failure Assessment) score to describe organ dysfunction/ failure. On behalf of the working group on sepsis-related problems of the european society of intensive care medicine. Intensive Care Med 22:707-710

47. Horby P, Lim WS, Emberson J, Mafham M, Bell J, Linsell L, Staplin N, Brightling C, Ustianowski A,Elmahi E, (2020) Dexamethasone in hospitalized patients with Covid-19_Preliminary Report. N Engl J Med. https://doi. org/10.1056/NEJMoa2021436

48. Müller B, Peri G, Doni A, Perruchoud AP, Landmann R, Pasqualini F, Mantovani A (2002) High circulating levels of the IL-1 type II decoy receptor in critically ill patients with sepsis: association of high decoy receptor levels with glucocorticoid administration. J Leukoc Biol 72:643-649

49. de Kruif MD, Lemaire LC, Giebelen IA, Struck J, Morgenthaler NG, Papassotiriou J, Elliott PJ, van der Poll T (2008) The influence of corticosteroids on the release of novel biomarkers in human endotoxemia. Intensive Care Med 34:518-522

50. Perren A, Cerutti B, Lepori M, Senn V, Capelli B, Duchini F, Domenighetti $G$ (2008) Influence of steroids on procalcitonin and C-reactive protein in patients with COPD and community-acquired pneumonia. Infection 36:163-166 
51. Rinaldi S, Adembri C, Grechi S, De Gaudio AR (2006) Low-dose hydrocortisone during severe sepsis: effects on microalbuminuria. Crit Care Med 34:2334-2339

52. Furst D, Saag K Glucocorticoid withdrawal. Uptodate. 2014 Mar [citado 25 Sep 2014]. In: Editor (eds) Book Glucocorticoid withdrawal. Uptodate. 2014 Mar [citado 25 Sep 2014]

53. Dinsen S, Baslund B, Klose M, Rasmussen AK, Friis-Hansen L, Hilsted L, Feldt-Rasmussen U (2013) Why glucocorticoid withdrawal may sometimes be as dangerous as the treatment itself. Eur J Internal Med 24:714-720

54. Schuetz $P$, Leuppi JD, Bingisser R, Bodmer M, Briel M, Drescher T, Duerring $U$, Henzen $C$, Leibbrandt $Y$, Maier S, Miedinger D, Mueller B, Scherr A, Schindler C, Stoeckli R, Viatte S, von Garnier C, Tamm M, Rutishauser $J$ (2015) Prospective analysis of adrenal function in patients with acute exacerbations of COPD: the Reduction in the Use of Corticosteroids in Exacerbated COPD (REDUCE) trial. Eur J Endocrinol 173:19-27

55. Hakkinen PJ, Schmoyer RL, Witschi HP (1983) Potentiation of butylatedhydroxytoluene-induced acute lung damage by oxygen. Effects of prednisolone and indomethacin. Am Rev Respir Dis 128:648-651

56. Kehrer JP, Klein-Szanto AJ, Sorensen EM, Pearlman R, Rosner MH (1984) Enhanced acute lung damage following corticosteroid treatment. Am Rev Respir Dis 130:256-261

57. Keh DBT, Weber-Cartens S, Schulz C, Ahlers O, Bercker S, Volk HD, Doecke WD, Falke KJ, Gerlach H (2003) Immunologic and hemodynamic effects of "low-dose" hydrocortisone in septic shock: a double-blind, randomized, placebo-controlled, crossover study. Am J Respir Crit Care Med 167:512-520

58. Gagnon S, Boota AM, Fischl MA, Baier H, Kirksey OW, La Voie L (1990) Corticosteroids as adjunctive therapy for severe Pneumocystis carinii pneumonia in the acquired immunodeficiency syndrome. A doubleblind, placebo-controlled trial. N Engl J Med 323:1444-1450

59. Nawab Q, Golden E, Confalonieri M, Umberger R, Meduri G (2011) Corticosteroid treatment in severe community-acquired pneumonia: duration of treatment affects control of systemic inflammation and clinical improvement. Intensive Care Med 37:1153-1554

60. Meduri GU, Bridges L, Siemieniuk RAC, Kocak M (2018) An exploratory reanalysis of the randomized trial on efficacy of corticosteroids as rescue therapy for the late phase of acute respiratory distress syndrome. Crit Care Med 46:884-891

61. Administration TFaD (Revised October 2011) SOLU-MEDROL ${ }^{\circledR}{ }^{(m e t h y l-}$ prednisolone sodium succinate for injection, USP). In: Editor (ed)^(eds) Book SOLU-MEDROL ${ }^{\circledR}$ (methylprednisolone sodium succinate for injection, USP). pp. 1-18

62. Amrein K, Oudemans-van Straaten HM, Berger MM (2018) Vitamin therapy in critically ill patients: focus on thiamine, vitamin C, and vitamin D. Intensive Care Med 44:1940-1944

63. Moskowitz A, Andersen LW, Huang DT, Berg KM, Grossestreuer AV, Marik PE, Sherwin RL, Hou PC, Becker LB, Cocchi MN, Doshi P, Gong J, Sen A, Donnino MW (2018) Ascorbic acid, corticosteroids, and thiamine in sepsis: a review of the biologic rationale and the present state of clinical evaluation. Crit Care 22:283

64. Fowler AA 3rd, Truwit JD, Hite RD, Morris PE, DeWilde C, Priday A, Fisher B, Thacker LR 2nd, Natarajan R, Brophy DF, Sculthorpe R, Nanchal R, Syed A, Sturgill J, Martin GS, Sevransky J, Kashiouris M, Hamman S, Egan KF, Hastings A, Spencer W, Tench S, Mehkri O, Bindas J, Duggal A, Graf J, Zellner S, Yanny L, McPolin C, Hollrith T, Kramer D, Ojielo C, Damm T, Cassity E, Wieliczko A, Halquist M (2019) Effect of vitamin C infusion on organ failure and biomarkers of inflammation and vascular injury in patients with sepsis and severe acute respiratory failure: the CITRIS-ALI Randomized Clinical Trial. JAMA 322:1261-1270

65. Takano K, Yamamoto S, Tomita K, Takashina M, Yokoo H, Matsuda N, Takano Y, Hattori Y (2011) Successful treatment of acute lung injury with pitavastatin in septic mice: potential role of glucocorticoid receptor expression in alveolar macrophages. J Pharmacol Exp Ther 336:381-390

66. Komatsubara M, Hara T, Hosoya T, Toma K, Tsukamoto-Yamauchi N, Iwata N, Inagaki K, Wada J, Otsuka F, (2017) Melatonin regulates catecholamine biosynthesis by modulating bone morphogenetic protein and glucocorticoid actions. J Steroid Biochem Mol Biol 165:182-189. https://doi. org/10.1016/j.jsbmb.2016.06.002
67. Hong J, Guo W-z, Yan Z-h, Di L, Lu C-I (2010) Influence of drug treatment on glucocorticoid receptor levels in patients with coronary heart disease. Chin Med J 123:1685-1689

68. Mantzarlis K, Tsolaki V,Zakynthinos E, (2017) Role of oxidative stress and mitochondrial dysfunction in sepsis and potential therapies. Oxid Med Cell Longev 2017:5985209. https://doi.org/10.1155/2017/5985209

69. Souffriau J, Libert C, (2018) Mechanistic insights into the protective impact of zinc on sepsis. Cytokine Growth Factor Rev 39:92-101. https:// doi.org/10.1016/j.cytogfr.2017.12.002

70. Erol N, Saglam L, Saglam YS, Erol HS, Altun S, Aktas MS,Halici MB, (2019) The protection potential of antioxidant vitamins against acute respiratory distress syndrome: a rat trial. Inflammation 42(5):1585-1594. https://doi. org/10.1007/s10753-019-01020-2

71. Siemieniuk RA, Meade MO, Alonso-Coello P, Briel M, Evaniew N, Prasad M, Alexander PE, Fei Y, Vandvik PO, Loeb M, Guyatt GH (2015) Corticosteroid therapy for patients hospitalized with community-acquired pneumonia: a systematic review and meta-analysis. Ann Intern Med 163:519-528

72. Arabi MY, Meduri GU, Chrousos GP, (2020) The ten reasons why corticosteroid therapy also reduces mortality in severe COVID-19. Intensive Care Med. https://doi.org/10.1007/s00134-020-06223-y

73. Lamontagne F, Agoritsas T, Macdonald H, Leo Y-S, Diaz J, Agarwal A, Appiah JA, Arabi Y, Blumberg L, Calfee CS, (2020) A living WHO guideline on drugs for covid-19. BMJ 370:m3379. https://doi.org/10.1136/bmj. m3379

74. Meduri GU, Schwingshackl A, Hermans G (2016) Prolonged glucocorticoid treatment in ARDS: impact on intensive care unit-acquired weakness. Front Pediatr 4:69

75. Meduri GU (2002) Clinical review: a paradigm shift: the bidirectional effect of inflammation on bacterial growth. Clinical implications for patients with acute respiratory distress syndrome. Crit Care 6:24-29

76. Kaufmann I, Briegel J, Schliephake F, Hoelzl A, Chouker A, Hummel T, Schelling G, Thiel M (2008) Stress doses of hydrocortisone in septic shock: beneficial effects on opsonization-dependent neutrophil functions. Intensive Care Med 34:344-349

77. Spencer-Segal JL, Hyzy RC, Iwashyna TJ, Standiford TJ (2017) Psychiatric symptoms in survivors of acute respiratory distress syndrome. Effects of age, sex, and immune modulation. Ann Am Thorac Soc 14:960-967

78. Schelling G, Briegel J, Roozendaal B, Stoll C, Rothenhausler HB, Kapfhammer HP (2001) The effect of stress doses of hydrocortisone during septic shock on posttraumatic stress disorder in survivors. Biol Psychiatry 50:978-985

79. Reddy K, Sinha P, O'Kane CM, Gordon AC, Calfee CS, McAuley DF (2020) Subphenotypes in critical care: translation into clinical practice. Lancet Respir Med 8:631-643

80. Meduri GU, Muthiah MP, Carratu P, Eltorky M, Chrousos GP (2005) Nuclear factor-kappaB- and glucocorticoid receptor alpha- mediated mechanisms in the regulation of systemic and pulmonary inflammation during sepsis and acute respiratory distress syndrome. Evidence for inflammation-induced target tissue resistance to glucocorticoids. NeurolmmunoModulation 12:321-338 